Check for updates

Cite this: Mater. Chem. Front., 2021, 5, 2900

Received 24th December 2020, Accepted 8th February 2021

DOI: 10.1039/d0qm01091

rsc.li/frontiers-materials

\section{Exploring molecular motors $\dagger$}

\author{
Jos C. M. Kistemaker $\ddagger^{a}$ Anouk S. Lubbe (D) and Ben L. Feringa (D) *ab
}

\begin{abstract}
The introduction of mechanical functions and controlled motion based on molecular motors and machines offers tremendous opportunities towards the design of dynamic molecular systems and responsive materials. In this brief perspective the focus is on the exploration of rotary molecular motors discussing basic principles and showing illustrative applications in functional materials. Furthermore, major challenges to bring dynamic properties to more complex molecular systems, ranging from transmission, amplification and synchronization to biocompatibility and autonomous behavior, are discussed.
\end{abstract}

Looking at Nature we witness an amazing collection of biological molecular motors and machines operating at the nanoscale, that may easily pass unnoticed until we see its macroscopic outcome - an organism moving. Analogous to macroscopic engines, Nature's molecular motors convert (photo-)chemical or thermal energy into mechanical motion and sustain an astonishing variety of dynamic functions in biomaterials and complex biological systems. Following this major source of inspiration for scientists in their endeavor to design synthetic molecular motors that can tolerate a wide range of structures, conditions and functions in materials well beyond natural systems, impressive progress towards artificial molecular machines has been made in the past decades. ${ }^{1-7}$ Facing the challenge to harness motion at the nanoscale to perform specific (mechanical) functions one should not underestimate random thermal Brownian motion, or, as described by Whitesides, "navigators on the nanoscale would have to accommodate to the Brownian storms that would crash against their hulls". 8 A molecular motor according to the general dictionary definition is anything "consisting of molecules" "that imparts motion", but to distinguish genuine molecular motors from e.g. conformational changes a more specific identification such as "an initial source of motive power designed to drive machinery" sounds more appropriate. ${ }^{9}$ Distinct from merely

\footnotetext{
${ }^{a}$ Stratingh Institute for Chemistry, University of Groningen, Nijenborgh 4, 9747 AG, Groningen, The Netherlands. E-mail: b.l.feringa@rug.nl

${ }^{b}$ Key Laboratory for Advanced Materials and Joint International Research Laboratory of Precision Chemistry and Molecular Engineering, Feringa Nobel Prize Scientist Joint Research Center, School of Chemistry and Molecular Engineering, East China University of Science and Technology, Shanghai, 200237, China $\dagger$ Dedicated to Professor J. P. Sauvage on the occasion of the 1st Sauvage Symposium at Wuhan University, 2020.

\$ Current address: Centre for Organic Photonics \& Electronics, School of Chemistry and Molecular Biosciences, The University of Queensland, Queensland 4072, Australia.
}

molecular switching, the repetitive and progressive nature of the motor's rotatory or translational movement should also be emphasized. Furthermore, with the future perspective to move chemistry into the realm of far-from-equilibrium behavior reminiscent of biological systems, autonomously operating molecular motors (besides catalytic systems) offer tremendous opportunities. With the advent of molecular nanoscience, gaining momentum after Feynman's wake-up call "There is plenty of room at the bottom" and taking advantage of the spectacular achievements in supramolecular chemistry and imaging techniques, among others, various molecular motors and machines exhibiting rotary and translational motion have been designed. ${ }^{10}$ A comparison between conventional macromotors and nano-size motors spanning dimensions from $26 \mathrm{~nm}^{11}$ (for biological motors) down to less than $1 \mathrm{~nm}^{12}$ (for synthetic motors) (Fig. 1), however, goes awkward in the nano-world of low Reynolds numbers where Brownian motion rules. ${ }^{13}$

Several features can be identified which characterize the properties of motors, including (i) the type of motion produced, (ii) "fuel" consumed and turned into movement, (iii) directionality achieved, (iv) turnover required i.e. repetitive, progressive behavior, and (v) autonomy of motor function. Rotational and translational motion are common types of motion, however, the distinction is sometimes less obvious as exemplified by the difference between light-driven overcrowded alkene rotary motors (Fig. 1e) ${ }^{18}$ and chemical fueled [2]catenane rotary motors (Fig. 1g). ${ }^{15}$ In the first case the motor rotary axle is intrinsic to the molecular structure whereas in the second system a small macrocycle is able to move translationally between stations on a larger macrocycle, resulting in an overall circular motion.

Table 1 summarizes the key properties of some of the rotary motors shown in Fig. 1, also in a historical perspective, with a focus on type of fuel, autonomous behavior and source of directionality. The emphasis in this brief perspective is on light-powered rotary 


\section{a}

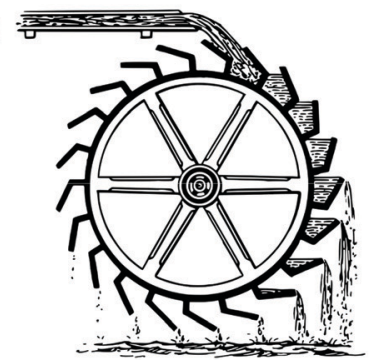

d

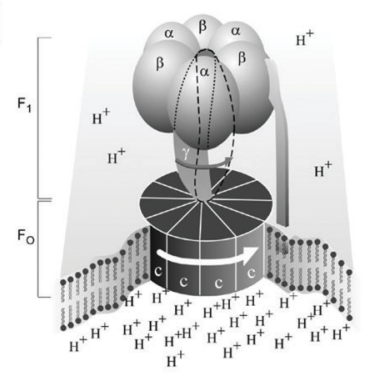

g

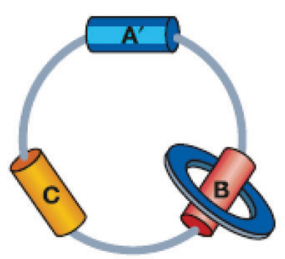

b

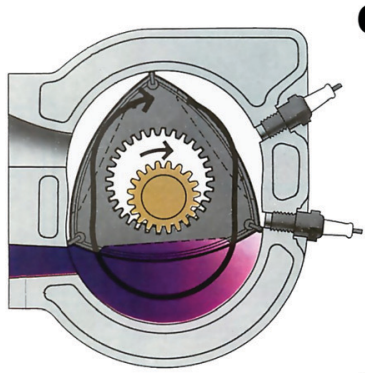

e

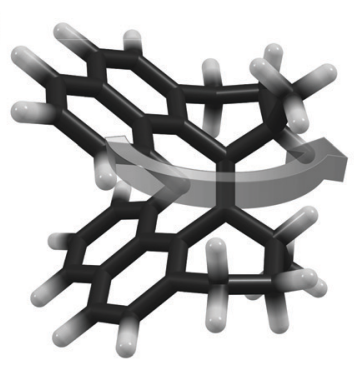

h

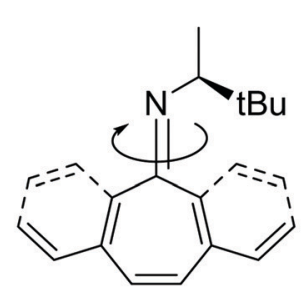

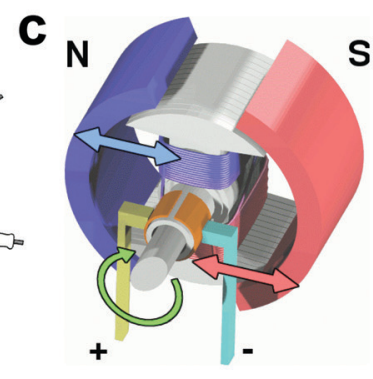

f
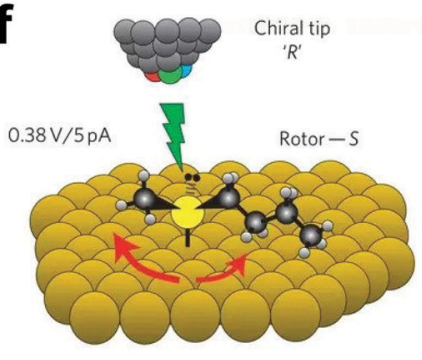

i

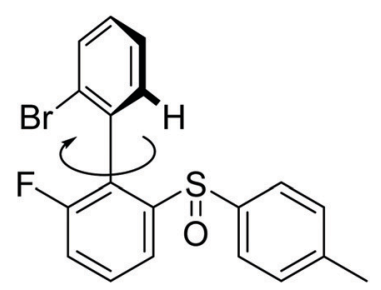

Fig. 1 Examples of rotary motors. (a) Water wheel, (b) Wankel internal combustion engine, (c) electric motor, (d) ATP synthase (reprinted from ref. 14), (e) molecular motor based on overcrowded alkene, (f) butyl methyl sulfide on copper surface driven by STM tip (adapted with permission from ref. 12, copyright 2011, Nature Publishing Group), (g) [2] catenane rotary motor (adapted with permission from ref. 15, copyright 2003, Nature Publishing Group), (h) imine-based molecular motor, ${ }^{16}$ (i) chemical driven rotary molecular motor. ${ }^{17}$

Table 1 Properties of rotary motors present in Fig. 1 (A indicates autonomy of the motor over its own fuel use or directionality)

\begin{tabular}{|c|c|c|c|c|c|}
\hline & Turnover (rpm) & Fuel & A & Directional source & A \\
\hline a & $2.0 \times 10^{1}$ & Water current & 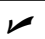 & Water flow & $x$ \\
\hline b & $7.0 \times 10^{3}$ & Combustion & $x$ & 2D asymmetry & $\boldsymbol{\nu}$ \\
\hline $\mathrm{c}$ & $1.0 \times 10^{5}$ & Electric current & レ & Electric flow & $x$ \\
\hline d & $8.0 \times 10^{3}$ & Proton gradient/ATP & レ & Chirality & $\boldsymbol{\nu}$ \\
\hline $\mathrm{e}$ & $1.8 \times 10^{8}$ & Light & $\nu$ & Chirality & $\nu$ \\
\hline $\mathrm{f}$ & $1.8 \times 10^{2}$ & Electric current & レ & Chirality & $x$ \\
\hline $\mathrm{g}$ & $6.9 \times 10^{-4}$ & Light & $x$ & Operator & $x$ \\
\hline $\mathrm{h}$ & Not reported & Light & $\boldsymbol{\nu}$ & Chirality & $\boldsymbol{\nu}$ \\
\hline $\mathrm{i}$ & $2.3 \times 10^{-4}$ & Chemical & $x$ & Chirality & $\boldsymbol{\nu}$ \\
\hline
\end{tabular}

motors but it should be noted that an interesting category pertains to Brownian motors operating for instance by information ratchet mechanisms. Also, the quest for control of directionality and the need for an operator versus autonomous behavior have been discussed elsewhere, in the context of chemical fueled rotary motors. $5,13,17$

Comparing a photochemical driven rotary motor (Fig. 2a) with a chemical driven rotary motor (Fig. 2b), the distinct operational mechanism is illustrated. ${ }^{19,20}$ In the autonomous light-powered motor (double bond rotary axle) the $360^{\circ}$ unidirectional rotation consist of a four step cycle comprising two photochemical driven $E-Z$ isomerizations each followed by a rate determining thermal isomerization. The two photochemical steps are energetically uphill while the thermal helix inversion steps are downhill in energy which, together with the chiral elements in the structure, is essential for the directionality of the rotary motion. In the biaryl-based chemical driven ratchet type motor (single bond rotary axle) again a four step rotation is involved. In contrast to the light-driven systems, a chiral source in the form of asymmetric catalysis allows for stereoselective cleavage of the lactone and control over directionality. Random rotation around the single bond is restricted due to steric effects and a sequence of selective protection/deprotection step and a repetition of these steps using appropriate chemical fuels completes a full, albeit non-autonomous, rotary cycle. Meanwhile, focusing on improving motion and dynamic functions, information ratchet mechanism based "molecular walkers", ${ }^{21}$ molecular pumps $^{22}$ and various chemical fueled rotary motors, including autonomous systems, metal catalyzed motors and rotary motors with near perfect control of directionality have been reported. ${ }^{17,20,23}$

Light-driven rotary motors have frequently have been employed as multistate chiral switches for instance in multifunctional chiral catalysts capable to deliver each enantiomer or the racemic version of a chiral product in an asymmetric transformation. ${ }^{24,25}$ A major advantage of the use of light as fuel or trigger signal is seen in materials applications where the fine-tuning of the energy and high spatial-temporal precision 
a

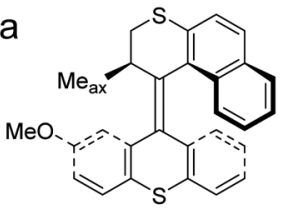<smiles>CCCCCCC</smiles>

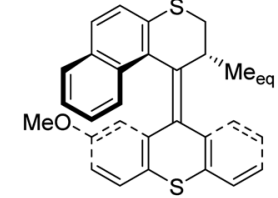<smiles>CC1CCCCC1</smiles><smiles></smiles>
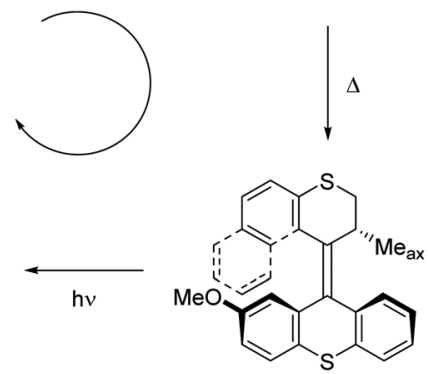

Fig. 2 Rotary cycles for (a) a photochemical driven motor and (b) a chemical driven motor.

can be particularly beneficial. Major steps to responsive, adaptive and non-equilibrium materials and functional systems include the incorporation of motors in polymers, ${ }^{26,27}$ liquid crystals $^{28,29}$ and supramolecular systems ${ }^{30-33}$ and the organization in porous solids ${ }^{34}$ and on surfaces. ${ }^{35-37}$ Pertinent examples shown in Fig. 3 illustrate various ways to achieve organization of rotary motors in 1D, 2D and 3D, amplification of motion, control of surface properties and helical organization and muscle function. b
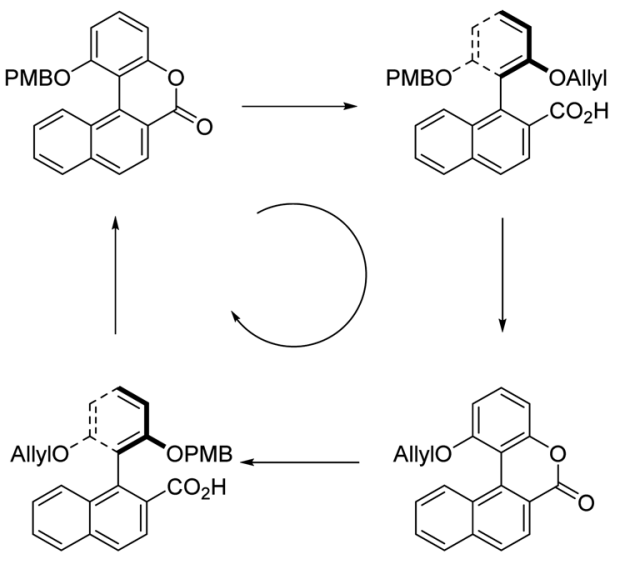

Transmission of motion from the molecular scale via mesoscopic to micro-scale was achieved using molecular rotary motors as chiral dopants in liquid crystal (LC) films (Fig. 3a). ${ }^{28}$ The change in helical chirality during a light-driven rotary cycle is amplified via a change in twisted nematic phase to modulate the structure and reflection color of the soft LC surface and allows to spin a microobject on top of the film. This interplay of light, molecular motion and mesoscopic organization offers attractive prospects for actuation and soft surface control. In a different approach rotary motors

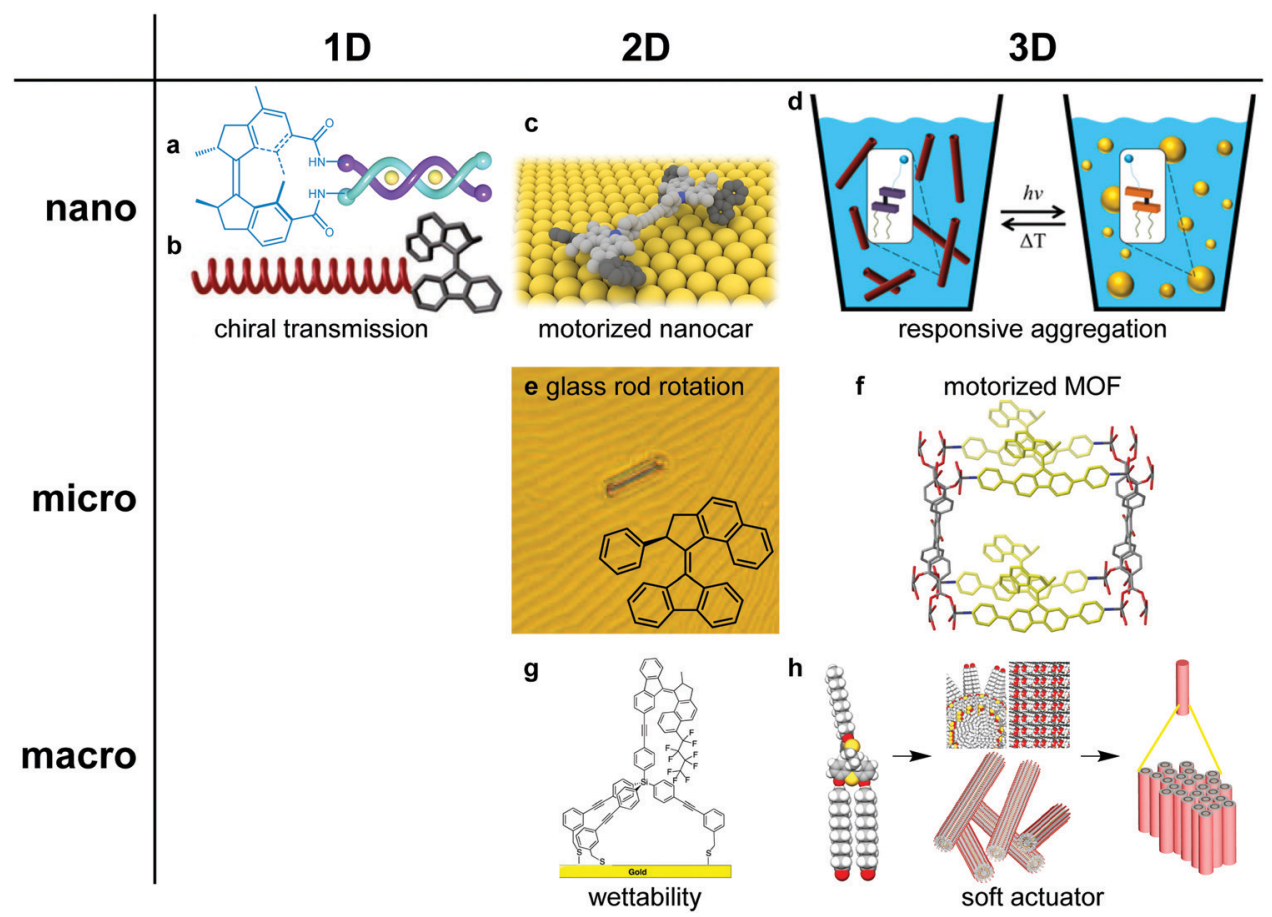

Fig. 3 Organization of rotary motors in one, two and three dimensions. (a) Molecular motor functionalized supramolecular Cu(I) helicate, (b) molecular motor controlling the twist sense of polyisocyanates, (c) motorized nanocar on a copper surface, (d) amphiphilic molecular motors forming responsive nanoscale assemblies in water, (e) liquid crystal doped with molecular motor, (f) motorized metal organic framework, (g) molecular motor functionalized gold surface, for control of wettability, and (h) artificial muscle built up by hierarchical self-assembly of amphiphilic molecular motor. Figures adapted with permission from ref. 27 (Copyright 2007, Wiley-VHC), 28 (Copyright 2006, Nature Publishing Group), 30 (Copyright 2016, Nature Publishing Group), 34 (Copyright 2019, Nature Publishing Group), 37 (Copyright 2014, American Chemical Society), 38 (Copyright 2016, American Chemical Society), 39 (Copyright 2018, Nature Publishing Group) and 45 (Copyright 2011, Nature Publishing Group). 
were covalently attached to surfaces in altitudinal and azimuthal orientations enabling e.g. control over surface wettability with light (Fig. 3b). ${ }^{37}$ Recently this approach was also used at the biointerface enabling control of surface protein layers and as a consequence the fate of adhering stem cells. ${ }^{40}$ Furthermore, with a perspective towards biocompatible systems various water soluble systems were designed in particular based on amphiphilic molecular motors (Fig. 3c). ${ }^{38}$ Operating as multistate switches they allow transformation between distinct nanoscale assemblies in water, like vesicles and nanotubes, as well as control of foam properties. ${ }^{41}$

Inspired by Lehn's supramolecular double helicates, ${ }^{42}$ motors were decorated with oligopyridine ligands for $\mathrm{Cu}(\mathrm{I})$ binding. The modulation between oligomers ( $E$ form) and monomer helicates with distinct helicity ( $Z$ form) and a far-from-equilibrium responsive supramolecular system upon continuous irradiation was achieved (Fig. 3d). ${ }^{30}$ In an alternative approach photochemical motors were used as initiators for the formation of dynamic helical polymers e.g. polyhexylisocyanate. (Fig. 3e). ${ }^{27}$ Here the rotary motion induced helix reversals in the polymer by amplification of the chirality and induces a mechanical effect from the molecular to the macromolecular level. Embedding a second generation rotary motor as crosslinker unit in a polymer gel Giuseppone and co-workers elegantly demonstrated contraction of the material with light. ${ }^{26}$ Amplification of motion along various length scales, from the molecular to macroscopic level, was demonstrated with a small molecule amphiphilic motor (Fig. 3f). ${ }^{39}$ Here the combination of self-assembly and supramolecular organization in water allowed actuation and muscle like function of this motor based responsive material. Finally, the 3D organization of rotary motors in reticular porous materials like MOFs is an attractive way to ultimately achieve cooperative effects and responsive solid materials. (Fig. 3g). ${ }^{34,43}$ Rotary motor were embedded as pillars in MOFs and covalent aromatic frameworks allowing control of porosity and adsorption ${ }^{44}$ taking advantage of the responsive motor function. The precise positioning of numerous motors, the design of robust yet dynamic solid materials and the construction of dual function "motorized MOFs' that can both absorb visible light and use this energy to fuel the motor pillars represent important steps toward future responsive materials. ${ }^{43}$ Although we are still at the dawn of the era of molecular motors and machines, from the examples shown here it is evident that light-driven molecular motors can function at various length scales in both hard and soft media and sustain distinct mechanical functions.

The future to bring dynamic properties to more complex systems through the incorporation of molecular motor functions, especially using light-driven rotary motors as discussed here, is particular bright. Considering the perspectives for smart and responsive molecular materials akin to biological materials we realize that a new era has arrived. Both the rapid advances in the field of molecular motors and machines and the challenges ahead, if we are able to translate for instance the interactive, self-regulatory, mechanical and adaptive behavior of Nature's fascinating materials into artificial ones, are testimony to the amazing opportunities arising. But it should also be clear that in order to design such materials we face the multifaceted challenge of unifying molecular structure, autonomous motion, organization, responsive functions, multicomponent assemblies, interfaces, hierarchical levels and length scales, just to mention a few. To address this next level of complexity a few of the challenges and opportunities ahead are outlined:

- Organization: like the ATPase motor rotating in our cell membranes or the myosin motors translating along actin tracks, the proper organization of molecular motors in a multicomponent system will be key to sustain a specific mechanical function. This can range from soft materials like liquid crystals, polymers, gels, artificial membranes and supramolecular fibers with substantial flexibility to more rigid matrices including porous solids and reticular materials (MOFs, COFs) etc. with more precise positioning. The control of organization, the extent to which assembly governs mutual function, the ensemble dynamics, the effects of viscosity, polarity, non-covalent interactions, the delicate balance of rigidity and flexibility and the organization of multicomponent assemblies with uncompromised function, are just a few of the challenges ahead for the materials designer.

- Transmission: in order to engineer molecular machinelike functions, it will be essential to learn how to transmit motion, for instance coupled rotary motion or converting rotary motion into translational motion. Designing e.g., a molecular gearbox, crankshafts, walkers, coupled rotors but also responsive surfaces or macromolecules capable to transmit motion and induce coupled mechanical effects are mainly limited by the imagination of the molecular engineer. The recent development of molecular cars, ${ }^{45}$ contracting gels, ${ }^{26}$ synchronized rotor-motor systems ${ }^{46}$ and mechanical systems in which chirality transmission takes place over nanometer length scales ${ }^{27}$ such as liquid crystal films using molecular motors as dopants enabling transmission form molecular to mesoscopic and microscopic dimensions, ${ }^{28}$ control of double helicate formation ${ }^{32}$ and polymer twist sense ${ }^{29}$ or multiple stereo-elements to control for instance asymmetric catalysis ${ }^{47}$ illustrates numerous fascinating opportunities arising.

- Amplification: converting molecular motion as a result of motor function into a macroscopic actuator effect requires amplification along multiple length scales, all the way from sub-nano to macro. A fine interplay of structural parameters, dynamics, assembly and ultimate function is essential. Here the combination of dynamic molecular structure, precise balance of interactions (also with the medium or materials matrix), the use of LC mesoscopic or polymer materials and exploring transmission mechanisms and self-organization offer numerous possibilities to achieve cooperative effects amplifying motion and transmit triggering signals. It should be noted that in order to construct a mechanical device, cooperativity and amplification will usually be key. ${ }^{48,49}$

- Catalytic driven machines: an as yet largely unexplored approach pertains to catalysis for mechanical function, especially in view of the fact that biological motors and machines almost exclusively rely on catalytic conversions to sustain a plethora of complex dynamic systems. Although catalytic 
propulsion systems ${ }^{50}$ for instance converting hydrogen peroxide $^{51}$ or glucose $^{52}$ as fuels and chemical-driven catalytic rotary motors (vide supra) have been demonstrated, development towards catalytic control of dynamic materials properties and machine like functions is still in its infancy. The power of catalysis to amplify molecular signals, induce cascades of transformations and assemble materials or change their properties hints to numerous dynamic systems waiting to be discovered.

- Synchronization and cooperativity: arguably the amplification of pico-Newton forces to the level of macroscopic actuation i.e., soft robotic function, will require cooperativity of molecular motors or machines. Here again the fine balance of organization, molecular interactions, adaptive properties, but also robustness play a key role. Crucial to future dynamic systems, particularly evident in surface bound rotary motors and 3D porous responsive materials, is the synchronization of motors. In order to achieve maximal benefit of mechanical motion and cooperative effects the synchronization of numerous motors is still a major challenge. This offers among others prospects for non-equilibrium systems, molecular communication and amplification and micro-and macroscopic oscillators.

- Interfaces: as evident from membrane bound nano-motors or micro-electronic devices, assembly of motors on surfaces or operating at interfaces is an important aspect to arrive at machine like functions or responsive materials. Molecular rotary motors have been covalently bound to various materials to generate responsive surfaces to control e.g. wettability. ${ }^{37}$ Precise 2D positioning at the nanoscale, as shown with porous crystal surfaces, ${ }^{53}$ can be essential for instance using these systems as multistage switches for information storage and processing and optoelectronic devices. Adapting molecular machines in order to function at the liquid interface especially in water and embedded in membranes will be an important prerequisite for the application of molecular motors in biological systems. ${ }^{54} \mathrm{An}$ inherent challenge is how to deal with the mutual influence, i.e., change in opto-electronic properties, effect of density of packing on dynamic function, communication, transmission and transduction mechanisms.

- Redox based motors: a dream for the molecular motorist is to design a genuine redox driven motor. Despite the fact that STM-driven electrical stimulated translational ${ }^{45}$ and rotary molecular motor function ${ }^{55}$ has been demonstrated as well as redox motors based on catenanes and rotaxanes,${ }^{56}$ the development of an autonomous electric rotary motor would open tremendous opportunities for future molecular machines.

- Multifunctional motor systems: this aspect deserves specific attention as designing a molecular motor will be more and more focused towards specific dynamic and mechanical functions. Integration of motion and interactive function is essential to the programming of specific tasks. Illustrative examples in which multistage switching and mechanical performance enables responsive and adaptive functions include small molecule transport, ${ }^{57}$ sequence specific peptide synthesis ${ }^{58}$ and multitasking catalysts for asymmetric transformations. ${ }^{24,25}$ With a general shift in focus from synthesis of structure to synthesis of function the interplay of functions, robustness, responsiveness and multicomponent nature will likely play a major role in applications ranging from "molecular factories" 49 to smart materials. ${ }^{59}$

- Biocompatibility: the increasing demand to interface synthetic systems and materials with cells and tissues provides an additional challenge to operate molecular motors and machines in aqueous environment. Facing the quest how to move into water the molecular designer is not only experiencing the hydrophilicityhydrophobicity balance but also has to factor in stability in and compatibility with the natural system i.e. enzymes and metabolism, oligonucleotides, membranes. The design of visible light or near IR driven motors, using harmless irradiation, is also a major task. With the first examples of light-driven motors controlling cell behavior ${ }^{40}$ and penetrating cell membranes ${ }^{60}$ reported, the prospects for artificial molecular motors-machines to construct bio-hybrid systems, develop drug-delivery systems or bring functional motors into the cell for detection or to regulate cellular function look particularly bright.

- Autonomous operation: a key feature of protein based motor and machines in biological systems is autonomous operation. Here the light-driven rotary motors stand out as upon irradiation and provided the barriers for rotation are appropriate, they can undergo continuous mechanical movement and push a system far from equilibrium. Although an autonomous chemical driven catenane-based rotary system has been reported ${ }^{61}$ several catalytic propulsion systems using hydrogen peroxide ${ }^{50}$ or glucose ${ }^{52}$ as fuels look particular promising to enable autonomous operation. With the prospect of larger (supramolecular) systems to operate fully autonomously, allow continuous mechanical functions and show out-of-equilibrium behavior characteristic of life itself, a major challenge is to demonstrate autonomous and continuous motion with photochemical molecular motors in multicomponent systems.

While exploring molecular motors the road to the future offers tremendous opportunities for the molecular explorer who is adventurous enough to go well beyond the static molecule. As outlined above, moving from molecules to dynamic systems and responsive materials powered by molecular motors we face many fundamental challenges, such as organization, cooperativity, amplification and biocompatibility, just to mention a few, but the explorer can take confidence in the fact that all these features are in harmony in a typical biological system leaving motion uncompromised. Yes, we will have to learn how to design dynamic systems which show motion, adaptive, responsive and self-regulatory behavior and in particular can act autonomous and sustain multiple functions. We might not face an "easy ride" but I am convinced the materials chemistry frontier will not encounter "slow motion".

\section{Conflicts of interest}

There are no conflicts to declare. 


\section{References}

1 V. Balzani, A. Credi, F. Raymo and J. F. Stoddart, Artificial molecular machines, Angew. Chem., Int. Ed., 2000, 39, 3348-3391.

2 W. R. Browne and B. L. Feringa, Making molecular machines work, Nat. Nanotechnol., 2006, 1, 25-35.

3 From Non-Covalent Assemblies to Molecular Machines, ed. J.-P. Sauvage and P. Gaspard, Wiley-VCH Verlag $\mathrm{GmbH}$ \& Co. KGaA, Weinheim, Germany, 2010.

4 The Nature of the Mechanical Bond: From Molecules to Machines, ed. C. J. Bruns and J. F. Stoddart, Wiley-VCH Verlag GmbH \& Co. KGaA, Weinheim, Germany, 2016.

5 S. Kassem, T. van Leeuwen, A. S. Lubbe, M. R. Wilson, B. L. Feringa and D. A. Leigh, Artificial molecular motors, Chem. Soc. Rev., 2017, 46, 2592-2621.

6 F. Lancia, A. Ryabchun and N. Katsonis, Life-like motion driven by artificial molecular machines, Nat. Rev. Chem., 2019, 3, 536-551.

7 V. García-López, D. Liu and J. M. Tour, Light-activated organic molecular motors and their applications, Chem. Rev., 2020, 120, 79-124.

8 G. M. Whitesides, The once and future nanomachine, Sci. Am., 2001, 285, 78-83.

9 Merriam Webster, Molecular Motor, https://www.merriamwebster.com/dictionary/, retrieved 08-12-2020.

10 R. P. Feynman, There is pleanty of room at the bottom, Eng. Sci., 1960, 23, 22-36.

11 T. Kon, K. Sutoh and G. Kurisu, X-ray structure of a functional full-length dynein motor domain, Nat. Struct. Mol. Biol., 2011, 18, 638-642.

12 H. L. Tierney, C. J. Murphy, A. D. Jewell, A. E. Baber, E. V. Iski, H. Y. Khodaverdian, A. F. McGuire, N. Klebanov and E. C. H. Sykes, Experimental demonstration of a singlemolecule electric motor, Nat. Nanotechnol., 2011, 6, 625-629.

13 R. D. Astumian, Design principles for Brownian molecular machines: how to swim in molasses and walk in a hurricane, Phys. Chem. Chem. Phys., 2007, 9, 5067-5083.

14 Press release: The 1997 Nobel Prize in Chemistry, http:// www.nobelprize.org/nobel_prizes/chemistry/laureates/1997/ press.html, retrieved 16-12-2020.

15 D. A. Leigh, J. K. Y. Wong, F. Dehez and F. Zerbetto, Unidirectional rotation in a mechanically interlocked molecular rotor, Nature, 2003, 424, 174-179.

16 L. Greb and J.-M. Lehn, Light-driven molecular motors: Imines as 4-step or 2-step unidirectional rotors, J. Am. Chem. Soc., 2014, 136, 13114-13117.

17 B. S. L. Collins, J. C. M. Kistemaker, E. Otten and B. L. Feringa, A chemically powered unidirectional rotary molecular motor based on a palladium redox cycle, Nat. Chem., 2016, 8, 860-866.

18 M. K. J. ter Wiel, R. A. van Delden, A. Meetsma and B. L. Feringa, Increased speed of rotation for the smallest light-driven molecular motor, J. Am. Chem. Soc., 2003, 125, 15076-15086.

19 N. Koumura, E. M. Geertsema, A. Meetsma and B. L. Feringa, Light-driven molecular rotor: Unidirectional rotation controlled by a single stereogenic center, J. Am. Chem. Soc., 2000, 122, 12005-12006.

20 S. P. Fletcher, F. Dumur, M. M. Pollard and B. L. Feringa, A reversible, unidirectional molecular rotary motor driven by chemical energy, Science, 2005, 310, 80-82.

21 M. von Delius and D. A. Leigh, Walking molecules, Chem. Soc. Rev., 2011, 40, 3656-3676.

22 C. Cheng, P. R. McGonigal, S. T. Schneebeli, H. Li, N. A. Vermeulen, C. Ke and J. F. Stoddart, An artificial molecular pump, Nat. Nanotechnol, 2015, 10, 547-553.

23 Y. Zhang, Z. Chang, H. Zhao, S. Crespi, B. L. Feringa and D. Zhao, A chemically driven rotary molecular motor based on reversible lactone formation with perfect unidirectionality, Chem, 2020, 6, 2420-2429.

24 R. Dorel and B. L. Feringa, Photoswitchable catalysis based on the isomerisation of double bonds, Chem. Commun., 2019, 55, 6477-6486.

25 J. Wang and B. L. Feringa, Dynamic control of chiral space, Science, 2011, 331, 1429-1432.

26 Q. Li, G. Fuks, E. Moulin, M. Maaloum, M. Rawiso, I. Kulic, J. T. Foy and N. Giuseppone, Macroscopic contraction of a gel induced by the integrated motion of light-driven molecular motors, Nat. Nanotechnol, 2015, 10, 161-165.

27 D. Pijper and B. L. Feringa, Molecular transmission: controlling the twist sense of a helical polymer with a single light-driven molecular motor, Angew. Chem., Int. Ed., 2007, 46, 3693-3696.

28 R. Eelkema, M. M. Pollard, J. Vicario, N. Katsonis, B. S. Ramon, C. W. M. Bastiaansen, D. J. Broer and B. L. Feringa, Molecular machines: nanomotor rotates microscale objects, Nature, 2006, 440, 163.

29 T. Orlova, F. Lancia, C. Loussert, S. Iamsaard, N. Katsonis and E. Brasselet, Revolving supramolecular chiral structures powered by light in nanomotor-doped liquid crystals, Nat. Nanotechnol, 2018, 13, 304-308.

30 R. Kramer, J.-M. Lehn and A. Marquis-Rigault, Selfrecognition in helicate self-assembly: spontaneous formation of helical metal complexes from mixtures of ligands and metal ions, Proc. Natl. Acad. Sci. U. S. A., 1993, 90, 5394-5398.

31 T. van Leeuwen, G. H. Heideman, D. Zhao, S. J. Wezenberg and B. L. Feringa, In situ control of polymer helicity with a non-covalently bound photoresponsive molecular motor dopant, Chem. Commun., 2017, 53, 6393-6396.

32 D. Zhao, T. van Leeuwen, J. Cheng and B. L. Feringa, Dynamic control of chirality and self-assembly of doublestranded helicates with light, Nat. Chem., 2016, 9, 250-256.

33 A. S. Lubbe, Q. Liu, S. J. Smith, J. W. de Vries, J. C. M. Kistemaker, A. H. de Vries, I. Faustino, Z. Meng, W. Szymanski, A. Herrmann and B. L. Feringa, Photoswitching of DNA hybridization using a molecular motor, J. Am. Chem. Soc., 2018, 140, 5069-5076.

34 W. Danowski, T. van Leeuwen, S. Abdolahzadeh, D. Roke, W. R. Browne, S. J. Wezenberg and B. L. Feringa, Unidirectional rotary motion in a metal-organic framework, Nat. Nanotechnol., 2019, 14, 488-494. 
35 R. A. van Delden, M. K. J. ter Wiel, M. M. Pollard, J. Vicario, N. Koumura and B. L. Feringa, Unidirectional molecular motor on a gold surface, Nature, 2005, 437, 1337-1340.

36 G. T. Carroll, G. London, T. F. Landaluce, P. Rudolf and B. L. Feringa, Adhesion of photon-driven molecular motors to surfaces via 1,3-dipolar cycloadditions: effect of interfacial interactions on molecular motion, ACS Nano, 2011, 5, $622-630$.

37 K.-Y. Chen, O. Ivashenko, G. T. Carroll, J. Robertus, J. C. M. Kistemaker, G. London, W. R. Browne, P. Rudolf and B. L. Feringa, Control of surface wettability using tripodal light-activated molecular motors, J. Am. Chem. Soc., 2014, 136, 3219-3224.

38 D. J. van Dijken, J. Chen, M. C. A. Stuart, L. Hou and B. L. Feringa, Amphiphilic molecular motors for responsive aggregation in water, J. Am. Chem. Soc., 2016, 138, 660-669.

39 J. Chen, F. K. C. Leung, M. C. A. Stuart, T. Kajitani, T. Fukushima, E. van der Giessen and B. L. Feringa, Artificial muscle-like function from hierarchical supramolecular assembly of photoresponsive molecular motors, Nat. Chem, 2018, 10, 132-138.

40 Q. Zhou, J. Chen, Y. Luan, P. A. Vainikka, S. Thallmair, S. J. Marrink, B. L. Feringa and P. van Rijn, Unidirectional rotating molecular motors dynamically interact with adsorbed proteins to direct the fate of mesenchymal stem cells, Sci. Adv., 2020, 6, eaay2756.

41 S. Chen, F. K. C. Leung, M. C. A. Stuart, C. Wang and B. L. Feringa, Dynamic assemblies of molecular motor amphiphiles control macroscopic foam properties, J. Am. Chem. Soc., 2020, 142, 10163-10172.

42 J.-M. Lehn, A. Rigault, J. Siegel, J. Harrowfield, B. Chevrier and D. Moras, Spontaneous assembly of double-stranded helicates from oligobipyridine ligands and copper(I) cations: structure of an inorganic double helix, Proc. Natl. Acad. Sci. U. S. A., 1987, 84, 2565-2569.

43 W. Danowski, F. Castiglioni, A. S. Sardjan, S. Krause, L. Pfeifer, D. Roke, A. Comotti, W. R. Browne and B. L. Feringa, Visible-light-driven rotation of molecular motors in a dual-function metal-organic framework enabled by energy transfer, J. Am. Chem. Soc., 2020, 142, 9048-9056.

44 F. Castiglioni, W. Danowski, J. Perego, F. K. C. Leung, P. Sozzani, S. Bracco, S. J. Wezenberg, A. Comotti and B. L. Feringa, Modulation of porosity in a solid material enabled by bulk photoisomerization of an overcrowded alkene, Nat. Chem., 2020, 12, 595-602.

45 T. Kudernac, N. Ruangsupapichat, M. Parschau, B. Maciá, N. Katsonis, S. R. Harutyunyan, K.-H. Ernst and B. L. Feringa, Electrically driven directional motion of a four-wheeled molecule on a metal surface, Nature, 2011, 479, 208-211.

46 P. Štacko, J. C. M. Kistemaker, T. van Leeuwen, M. Chang, E. Otten and B. L. Feringa, Locked synchronous rotor motion in a $\mathrm{M} \backslash$ molecular motor, Science, 2017, 356, 964-968.
47 S. F. Pizzolato, P. Štacko, J. C. M. Kistemaker, T. van Leeuwen and B. L. Feringa, Phosphoramidite-based photoresponsive ligands displaying multifold transfer of chirality in dynamic enantioselective metal catalysis, Nat. Catal., 2020, 3, 488-496.

48 D. Dattler, G. Fuks, J. Heiser, E. Moulin, A. Perrot, X. Yao and N. Giuseppone, Design of collective motions from synthetic molecular switches, rotors, and motors, Chem. Rev., 2020, 120, 310-433.

49 S. Krause and B. L. Feringa, Towards artificial molecular factories from framework-embedded molecular machines, Nat. Rev. Chem., 2020, 4, 550-562.

50 K. K. Dey and A. Sen, Chemically propelled molecules and machines, J. Am. Chem. Soc., 2017, 139, 7666-7676.

51 C. Stock, N. Heureux, W. R. Browne and B. L. Feringa, Autonomous movement of silica and glass micro-objects based on a catalytic molecular propulsion system, Chem. Eur. J., 2008, 14, 3146-3153.

52 D. Pantarotto, W. R. Browne and B. L. Feringa, Autonomous propulsion of carbon nanotubes powered by a multienzyme ensemble, Chem. Commun., 2008, 1533-1535.

53 J. Kaleta, J. Chen, G. Bastien, M. Dračínský, M. Mašát, C. T. Rogers, B. L. Feringa and J. Michl, Surface inclusion of unidirectional molecular motors in hexagonal tris(ophenylene)cyclotriphosphazene, J. Am. Chem. Soc., 2017, 139, 10486-10498.

54 K. Ariga, The evolution of molecular machines through interfacial nanoarchitectonics: from toys to tools, Chem. Sci., 2020, 11, 10594-10604.

55 A. Saywell, A. Bakker, J. Mielke, T. Kumagai, M. Wolf, V. García-López, P.-T. Chiang, J. M. Tour and L. Grill, Light-induced translation of motorized molecules on a surface, ACS Nano, 2016, 10, 10945-10952.

56 M. Baroncini, S. Silvi and A. Credi, Photo- and redox-driven artificial molecular motors, Chem. Rev., 2020, 120, 200-268.

57 J. Chen, S. J. Wezenberg and B. L. Feringa, Intramolecular transport of small-molecule cargo in a nanoscale device operated by light, Chem. Commun., 2016, 52, 6765-6768.

58 B. Lewandowski, G. D. Bo, J. W. Ward, M. Papmeyer, S. Kuschel, M. J. Aldegunde, P. M. E. Gramlich, D. Heckmann, S. M. Goldup, D. M. D’Souza, A. E. Fernandes and D. A. Leigh, Sequence-Specific Peptide Synthesis by an Artificial SmallMolecule Machine, Science, 2013, 339, 189-193.

59 Q. Zhang, D.-H. Qu, H. Tian and B. L. Feringa, Bottom-up: can supramolecular tools deliver responsiveness from molecular motors to macroscopic materials?, Matter, 2020, 3, 355-370.

60 V. García-López, F. Chen, L. G. Nilewski, G. Duret, A. Aliyan, A. B. Kolomeisky, J. T. Robinson, G. Wang, R. Pal and J. M. Tour, Molecular machines open cell membranes, Nature, 2017, 548, 567-572.

61 M. R. Wilson, J. Solà, A. Carlone, S. M. Goldup, N. Lebrasseur and D. A. Leigh, An autonomous chemically fuelled small-molecule motor, Nature, 2016, 534, 235-240. 